\title{
Primary mesenteric adenocarcinoma covered by abscess of the mesocolon and intestinal obstruction: A case report
}

\author{
YUN LUO, MEMGCHUAN OU, YUSHUN ZHANG, ZHIMING FU and CHONGSHU WANG \\ Department of General Surgery, The Affiliated Hospital \\ of North Sichuan Medical College, Nanchong, Sichuan 637000, P.R. China
}

Received March 29, 2016; Accepted February 1, 2017

DOI: $10.3892 / \mathrm{mco} .2017 .1164$

\begin{abstract}
Primary mesenteric adenocarcinoma (PMA) is a rare malignant disease. A case of PMA that was covered by an abscess of the mesocolon and intestinal obstruction is reported in the present study. As the onset site of PMA is often obscured or hidden, there may be no clinical symptoms in the early stage. At the advanced stage, the tumor size is large enough to induce intestinal obstruction, perforation, bleeding, volvulus and other clinical symptoms in the majority of patients. Computed tomography scans, ultrasound and other auxiliary examinations are not sufficiently accurate for diagnosing PMA with non-specific characteristics in the majority of cases, causing certain difficulties in the pre-operative diagnosis of PMA, which easily lead to misdiagnosis. The main effective treatment of PMA is surgical resection as early as possible. Thus, when a patient is suspected of having malignancies, and gastrointestinal cancer and other malignancies of the digestive organs may be excluded, the possibility of PMA should be considered.
\end{abstract}

\section{Introduction}

Primary mesenteric adenocarcinoma (PMA) is a rare type of malignant tumor. A previous study has presented a case of embryonal carcinoma originating in the mesentery (1). A further case study reported on poorly differentiated adenocarcinoma of unknown primary site detected by mesenteric abscess formation (2). The present case study reports on a case of PMA that was covered by an abscess of the mesocolon, and had the complication of intestinal obstruction. The clinical data of the case are presented, the relevant

Correspondence to: Professor Chongshu Wang, Department of General Surgery, The Affiliated Hospital of North Sichuan Medical College, 234 Fujiang Road, Nanchong, Sichuan 637000, P.R. China E-mail: chongs-wang@163.com

Key words: primary mesenteric adenocarcinoma, abscess, intestinal obstruction literature has been reviewed, and the management of PMA is discussed.

\section{Case report}

First admission. A 49-year-old male patient was admitted to the Affiliated Hospital of North Sichuan Medical College (Nanchong, China) in February 2015 due to abdominal pain for $>7$ months, aggravated by nausea and no defecation for 6 days. Physical examination revealed a tenderness in the left side of the abdomen with bowel sound hyperfunction, and a digital rectal examination did not reveal any abnormalities. The white blood cell count was $12.96 \times 10^{9} / 1$. Analysis of tumor markers showed that the levels of carcinoembryonic antigen (CEA) and carbohydrate antigen (CA)19-9 were $14.33 \mu \mathrm{g} / \mathrm{l}$ (normal, 0-5 $\mu \mathrm{g} / \mathrm{l}$ ) and 757.1 U/ml (normal,0-37 U/ml), respectively. An abdominal X-ray plain film revealed intestinal obstruction (Fig. 1), and a computed tomography (CT) scan of the abdomen revealed an incomplete ileus (Fig. 2A) and low-density lesions in the spleen (Fig. 2B). The preliminary diagnosis of acute complete intestinal obstruction, and possibly of gastrointestinal carcinoma, was considered. Upon laparotomy, an abscess of the mesocolon of $\sim 6 \times 5 \times 4 \mathrm{~cm}$ in size was found below the splenic flexure of the colon, the transverse colon was expanded and the regions surrounding the intestinal wall had a lot of pus associated with edema. The left colon mesentery with surrounding tissue adhesion showed hyperemia, edema and hardness. After the abscess was resected, $20 \mathrm{ml}$ pus was obtained for bacterial culture and drug sensitivity testing. Biopsy of the full thickness of the abscess base and a qualitative biopsy of the hard mesentery revealed acute gangrenous inflammation (Fig. 3). Terminal ileum colostomy was subsequently performed, following which the patient recovered well after fighting infection and symptomatic treatment. Specifically, the infection was treated with Cefotiam antibiotics, and after 6 days of anti-infection treatment with Cefotiam, the patient's condition gradually improved; he was discharged from hospital after recovery.

Second admission. The patient was admitted to hospital again in September 2015 due to ileum colostomy closure. The serum concentrations of CEA and CA19-9 were elevated $(49.44 \mu \mathrm{g} / \mathrm{l}$ and $2,569 \mathrm{U} / \mathrm{ml}$, respectively), and compared with that on first admission, the CT scan showed that the left side of the 


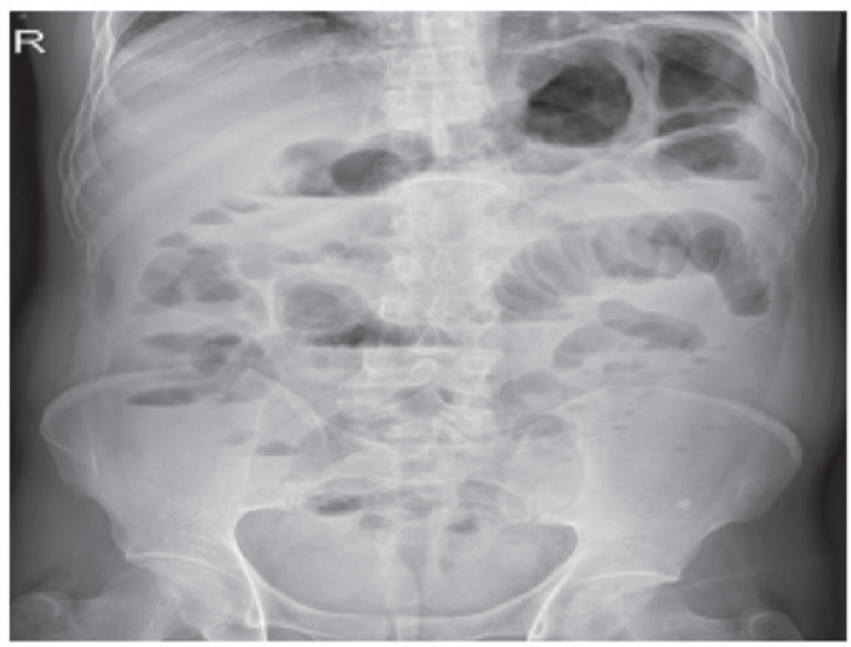

Figure 1. Abdominal X-ray plain film showing intestinal obstruction. The splenic flexure of the colon is most clearly expanded. R, right.

\section{A}

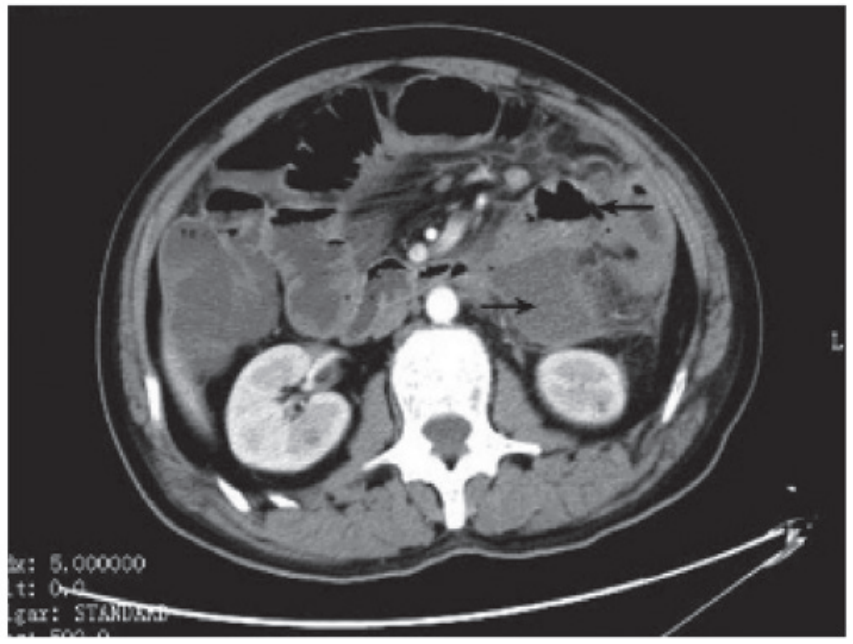

B

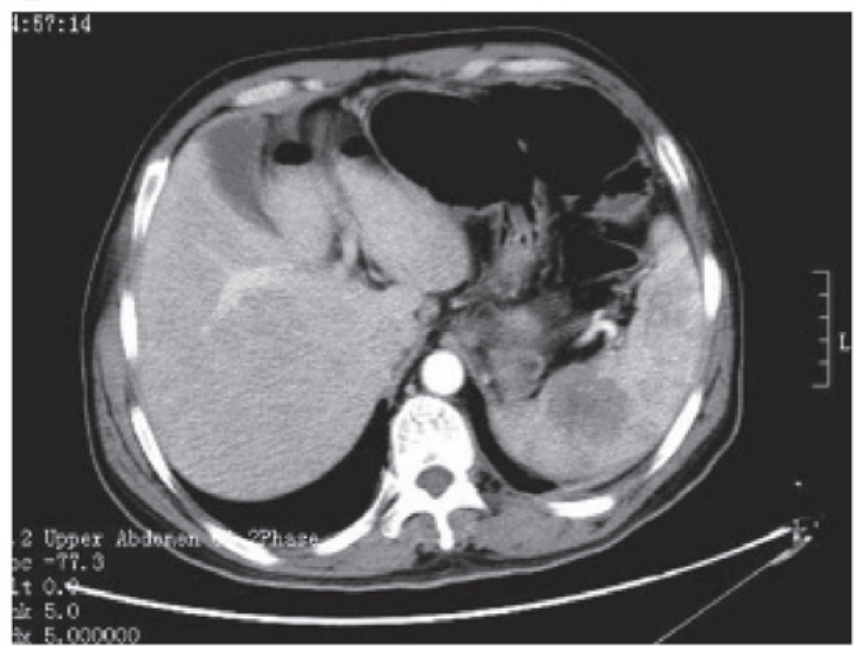

Figure 2.CT scan of the abdomen, and low-density lesions in the spleen. (A) CT image including the splenic flexure of the colon (indicated by $\leftarrow$ ), revealing the abscess of the mesocolon as an adjacent shadow (indicated by $\rightarrow$ ) and an unevenly thickened bowel wall. (B) Enhanced CT scan showing a low-density lesion of the spleen on first admission. CT, computed tomography.

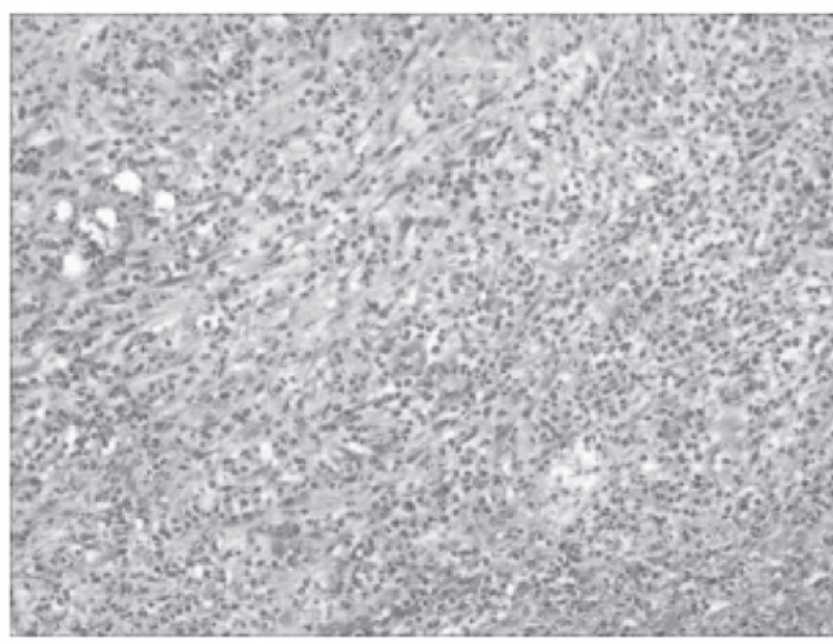

Figure 3. Hematoxylin and eosin staining, showing acute gangrenous inflammation of the abscess wall of the mesocolon (magnification, x100).

abdomen featured an irregular soft-tissue shadow (Fig. 4A) and the shadow of the lesions of the spleen was significantly increased (Fig. 4B). A second laparotomy was performed. Pale yellow ascites $(\sim 100 \mathrm{ml})$ were obtained. A mesenteric solid tumor of the colon of $\sim 8 \times 6 \times 6 \mathrm{~cm}$ in size was present near the left side of the superior mesenteric artery root, and the tumor, with qualitative hardness (hard texture), poor activity and unclear boundaries, infiltrated areas including the surrounding mesenteric vessels, small intestine and transverse colon. A hard mass with a diameter of $\sim 6 \mathrm{~cm}$ was palpitated in the spleen. There were no abnormal findings in the gastrointestinal tract, liver, pancreas, kidney and other organs. Three tissue samples of $\sim 0.5 \times 0.5 \times 0.5 \mathrm{~cm}$ in size from different parts of the mesenteric tumor were used for diagnosis based on intraoperative frozen section analysis, which revealed adenocarcinoma (Fig. 5). Owing to the difficulties in resecting the tumor, no further surgery was performed on the patient following communication with his family. During the 3 months of follow-up, the patient did not receive any adjuvant chemotherapy and his cancer cachexia worsened.

\section{Discussion}

Primary mesenteric tumors (PMT) are encountered in $\sim 1 / 10,000-1 / 25,000$ of the total number of hospitalized patients. In patients with PMT, the cystic/solid ratio was reported to be 2:1 and the former type was more common for benign tumors, while the latter type was mainly encountered in malignant cases (3). The World Health Organization lists 34 different pathological classifications for PMT, among which adenocarcinoma is a rare type. The present study reported on a unique and rare case of PMA, which was covered by an abscess of the mesocolon and featured intestinal obstruction.

As the onset site of PMT is often obscured or hidden, patients in the early stage have no clinical symptoms. Only when the tumor size is sufficiently large for it to burst or to exert effects including bowel compression or torsion of the mesentery, may it induce intestinal obstruction, perforation, bleeding, volvulus and secondary abdominal infections. Therefore, patients with 


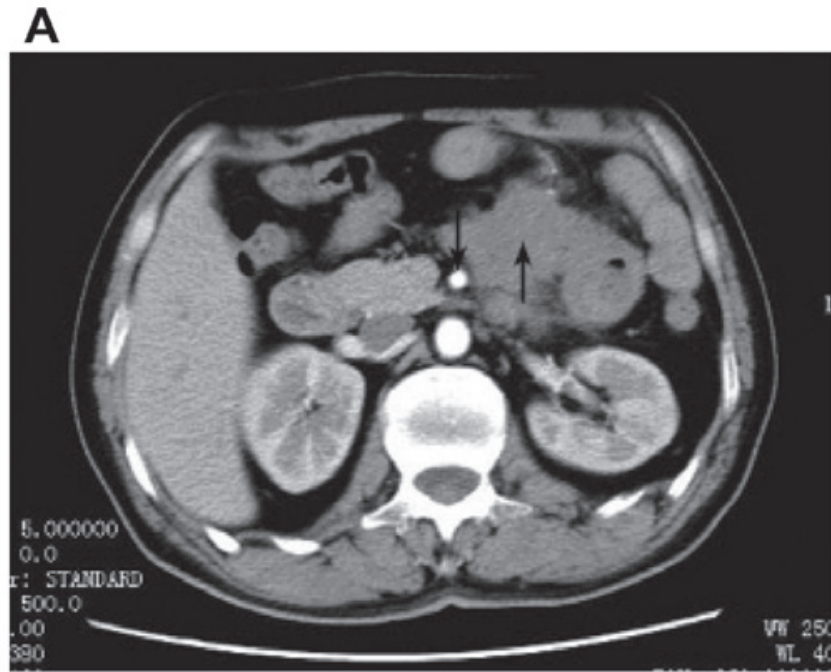

B

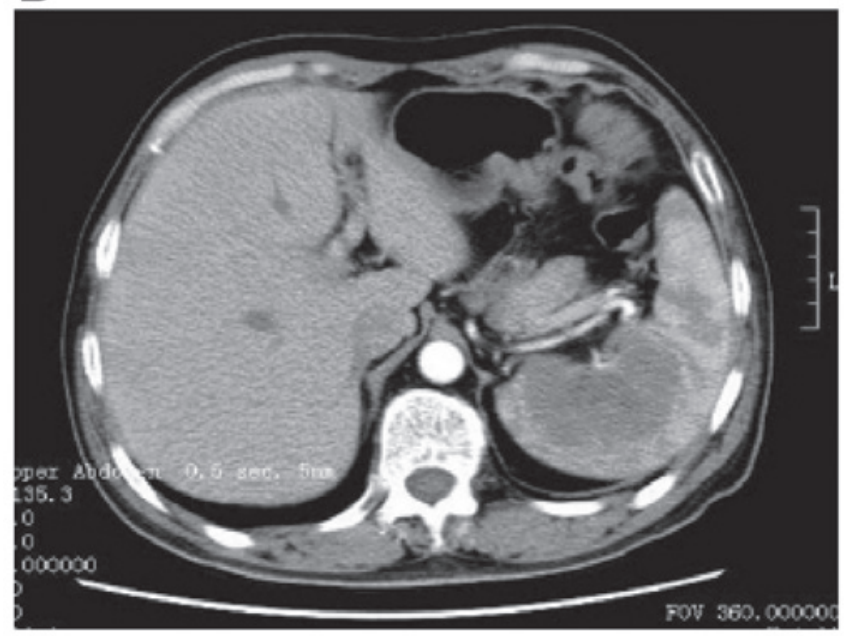

Figure 4. CT scan of the left side of the abdomen on second admission of the patient. (A) Mesenteric adenocarcinoma was shown as an irregular soft tissue shadow (indicated by $\uparrow$ ) near to the left side of the superior mesenteric artery (indicated by $\downarrow$ ). (B) CT image on second admission, showing that the shadow on the spleen was significantly increased compared with that on first admission. CT, computed tomography.

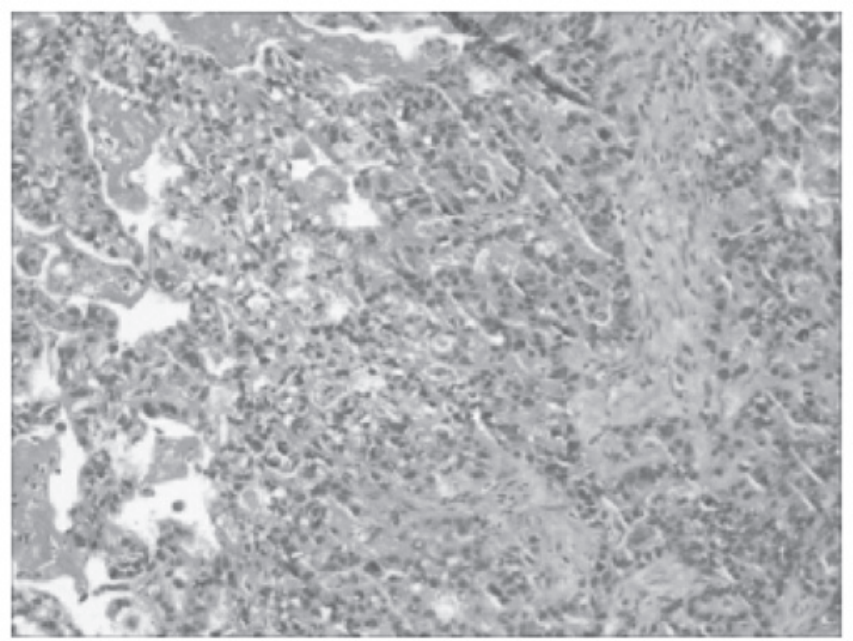

Figure 5. Intraoperative frozen section analysis of the mesenteric tumor during the second laparotomy, revealing poorly differentiated adenocarcinoma (hematoxylin and eosin staining; magnification, x200). clinical symptoms have reached the advanced stage with a pre-operative diagnosis rate of only 9.38-40.2\% (4). CT, ultrasound and other imaging techniques are able to provide a reference for tumor shape, size, borders, bleeding, breakage and metastasis. However, the clinical features of patients with PMT are complex. In CT images, tumors with certain characteristics can be identified prior to surgery, while other non-specific tumors can only be diagnosed by intraoperative frozen or post-operative pathological examination (5). At the first laparotomy of the present case, only an abscess of the mesocolon near the splenic flexure was identified, in which acute gangrenous inflammation was confirmed by biopsy. Abdominal abscesses concomitant with malignant tumors are more common in patients with gastrointestinal cancer associated with chronic perforation $(6,7)$; however, in the present case, no gastrointestinal tumor was present. Finally, the patient was diagnosed with an abscess of the mesocolon and intestinal obstruction, while PMA was overseen.

The pre-operative diagnosis of PMT is often challenging and affected patients are easily misdiagnosed for the following reasons: First, patients at early stages do not have any specific clinical symptoms. Furthermore, CT and other auxiliary examinations can localize the tumor, but cannot provide any qualitative diagnosis. When PMT invades or adheres to adjacent structures, unclear tumor boundaries may reduce the accuracy of CT localization and obstruct diagnosis. In addition, PMT is rare and clinicians are often not sufficiently aware of the disease. Finally, clinicians may be contented with the diagnosis of complications or comorbidities, and ignore PMT as the fundamental cause. In the present case, the patient with acute abdominal manifestation of intestinal obstruction was admitted, the pre-operative leucocyte count and tumor marker levels were high, CT indicated intestinal obstruction, and the intraoperative diagnosis was an abscess of the mesocolon with pathological examination revealing inflammation. As concomitant diseases were more apparent, the diagnosis of PMT was not considered.

Surgical resection is predominant as the most effective method for the treatment of PMT, with resection rates of benign and malignant tumors of 81.9 and $55.0 \%$, respectively. Most malignant tumors are located in the root of the mesentery and infiltrate major mesenteric blood vessels (8), which is the main reason for the low resection rates of malignant PMT. To predict whether patients with PMT may benefit from adjuvant radiation and chemotherapy, factors including the degree of malignancy, histological type and age may be considered.

In conclusion, the present study presented a rare and unique case of PMA, which was covered and misdiagnosed as an abscess of the mesocolon and intestinal obstruction. An increased understanding of PMT among clinicians may reduce the rate of misdiagnosis of PMT. When a patient is suspected of having malignancies, and gastrointestinal cancer and other malignancies of the digestive organs may be excluded, the possibility of PMA should be considered.

\section{References}

1. Ohshima T, Imada T, Nozawa A, Rino Y and Takanasi Y: A case of primary mesenteric embryonal carcinoma. Hepatogastroenterology 51: 983-986, 2004. 
2. Yamagata Y, Ando Y, Matsusaka K, Karube H, Onoyama H, Aikou S, Yamashita H, Mori K, Nomura S, Fukayama M and Seto Y: Poorly differentiated mesenteric carcinoma of unknown primary site detected by abscess formation: Case report. World J Surg Oncol 12: 4, 2014.

3. Shao-Yun C, Shi-De L and Hong X: The diagnosis and treatment of primary mesenteric tumor. Progr Mod Biomed 7: 1182-1183, 2007.

4. Cheng L: Diagnosis and treatment of primary intestinal mesentery tumor. Chin J Gen Surg 13: 395-396, 2004.

5. Cuiping Z, Wenfeng Z, Mingwei L, Zhihun Y and Danling N CT findings of primary mesenteric tumors. J Clin Radiol 31: $1131-1134,2012$
6. Chen HS and Sheen-Chen SM: Obstruction and perforation in colorectal adenocarcinoma: An analysis of prognosis and current trends. Surgery 127: 370-376, 2000.

7. Alcobendas F, Jorba R, Poves I, Busquets J, Engel A and Jaurrieta E: Perforated colonic cancer. Evolution and prognosis. Rev Esp Enferm Dig 92: 326-333, 2000.

8. $\mathrm{Ru} \mathrm{Z}$ : Research and progress of primary intestinal mesentery tumor. Med Rev 8: 278-279, 2002. 